\title{
Analyse statistique des moments d'ordre élevé dans une couche limite turbulente en présence de différences de densité importantes
}

\author{
Azeddine Soudani ${ }^{1, a}$ ET Rachid BessaÏH ${ }^{2}$ \\ 1 Département de physique, Faculté des Sciences, Université de Batna, Rue Chahid Mohamed El Hadi Boukhlouf, \\ Batna, Algérie \\ 2 Département de Génie Mécanique, Faculté des Sciences de l'Ingénieur, Université Mentouri-Constantine, Route d'Ain El Bey, \\ Constantine, Algérie
}

Reçu le 28 mars 2003, accepté le $1^{\text {er }}$ octobre 2003

\begin{abstract}
Résumé - Afin d'étudier la structure fine de la turbulence pariétale en présence de variations importantes de densité, une analyse statistique des données expérimentales, dans une soufflerie à recirculation, est effectuée. Les résultats montrent que la relaxation du facteur de dissymétrie de $u^{\prime}\left(S_{u^{\prime}}\right)$ s'effectue plus rapidement dans la couche externe que près de la paroi, aussi bien pour l'injection de l'air que pour l'injection d'hélium. $S_{u^{\prime}}$ croît près de la fente d'injection de façon appréciable et cette augmentation est nettement plus accentuée pour l'injection de l'air que l'injection d'hélium. Cette croissance du facteur de dissymétrie près de la fente d'injection peut s'expliquer par l'augmentation du flux convectif longitudinal de l'énergie turbulente dans cette zone. Les résultats montrent, pour la distribution du facteur d'aplatissement $F_{u^{\prime}}$, qu'il n'y a pas d'effet important du gradient de densité sur la structure intermittente de la vitesse longitudinale instantanée dans la zone développée, $x / \delta \geq 5$. L'analyse statistique menée dans cette étude montre que l'injection d'hélium, dans la couche limite, engendre des éjections plus violentes que dans le cas d'une injection d'air. Ce résultat est confirmé par la contribution importante des éjections au flux massique turbulent.
\end{abstract}

Mots clés : Couche limite turbulente / structures cohérentes / mélange de gaz / densité variable / éjection / balayage

Abstract - Statistical analysis of high order moments is a turbulent boundary layer with strong density differences. In order to study the fine structure of wall turbulence in the presence of significant variations of density, a statistical analysis of the experimental data, obtained in a wind tunnel, is carried out. The results show that the relaxation of the skewness factor of $u^{\prime}\left(S_{u^{\prime}}\right)$ is carried out more quickly in the external layer than close to the wall, as well for the air injection as for the helium injection. $S_{u^{\prime}}$ grows close to the injection slot in an appreciable way and this increase is accentuated for the air injection than for the helium injection. This growth of the skewness factor close to the injection slot can be explained by the increase in the longitudinal convective flux of turbulent energy in this zone. The results show for the distribution of the flatness factor $F_{u^{\prime}}$ that there is no significant effect of the density gradient on the intermittent structure of the instantaneous longitudinal velocity in the developed zone, $x / \delta \geq 5$. The statistical analysis carried out in this study shows that the helium injection in the boundary layer generates more violent ejections than in the case of air injection. This result is confirmed by the significant contribution of the ejections to turbulent mass flux.

Key words: Turbulent boundary layer / coherent structures / gas mixture / variable density / ejections / sweeps

\footnotetext{
a Auteur correspondant :

soudani.azeddine@caramail.com
} 


\section{Introduction}

L'étude des couches limites turbulentes en présence de fortes différences de densité est d'une importance fondamentale dans la compréhension des différents phénomènes qui entrent en jeu dans des situations pratiques importantes, comme en combustion, lors de l'entrée dans l'atmosphère où les différences importantes de température entre la paroi et la couche externe donnent lieu à de fortes variations de masse volumique, ou encore lors des refroidissements des pales de turbines par injection tangentielle d'un gaz différent. Le sujet a également son importance, notamment dans la compréhension de l'influence des différences de densité sur les propriétés du transport turbulent. Des travaux ont déjà essayé d'apporter des informations détaillées concernant les quantités turbulentes dans ce type d'écoulements [1-5], mais plusieurs problèmes restent à éclaircir.

L'objectif de la présente étude est d'éclaircir l'effet de la variation de densité à partir des moments d'ordre trois (facteur de dissymétrie) et celui d'ordre quatre (facteur d'aplatissement) des fluctuations de vitesse et de densité d'une part et d'autre part de contribuer à l'étude de la structure de la turbulence pariétale en présence de variations importantes de densité, en analysant l'effet de ces différences de densité sur les changements qui peuvent arriver dans la structure des grandes échelles et sur les mécanismes du mouvement du fluide, en analysant les données expérimentales obtenues par Harion [2] dans une soufflerie à recirculation. Plusieurs types de structures ont pu être mis en évidence dans une couche limite turbulente homogène, en particulier à partir des expériences de visualisation. Néanmoins, afin d'obtenir des informations plus quantitatives, ces structures sont généralement identifiées à partir d'analyses statistiques conditionnelles de signaux de fluctuations de vitesse, de vorticité, ou encore de la température de l'écoulement [6-11]. Les événements sont dits cohérents à partir de critères, généralement fondés sur la durée et l'intensité de certaines fluctuations. Ces critères sont souvent définis de manière à retrouver les fréquences d'apparition de ces événements données par les expériences de visualisation [12-16]. Parmi les événements cohérents largement étudiés en couche limite turbulente, les balayages («sweeps »), les éjections et les «bursts » ont fait l'objet d'une large part des préoccupations depuis la fin des années soixante. Les balayages constituent des mouvements cohérents de fluide venant des régions extérieures de la couche limite et se dirigeant vers la paroi à grande vitesse. Au contraire, les éjections sont les mouvements du fluide à faible vitesse en direction de l'écoulement externe. Quant au terme de « burst », il caractérise un ensemble d'éjections successives regroupées à l'intérieur d'un même événement [12]. De plus, le processus de « bursting » indique l'ensemble du cycle balayageséjections qui se répète de façon relativement régulière. Il est montré qu'au cours de ces cycles une partie importante de l'énergie cinétique turbulente est produite dans la couche limite [17-19]. Dans notre étude, nous avons opté pour la représentation des moments d'ordre trois (facteur de dissymétrie) et celui d'ordre quatre (facteur

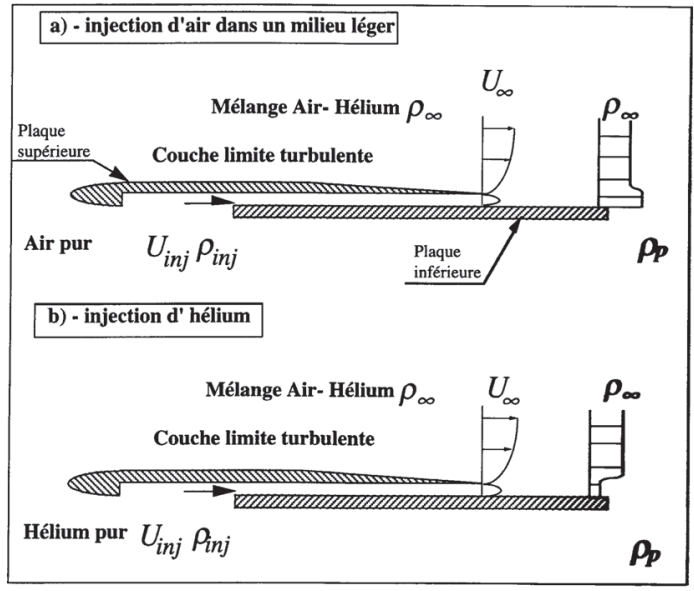

Fig. 1. Principe de l'expérience.

d'aplatissement), car elle permet de prendre en compte la distribution temporelle de $\rho^{\prime}(t)$, qui pour $u^{\prime}(t)<0$ ou $u^{t}(t)>0$ donnée, résulte respectivement en $\rho^{\prime}(t) \partial \bar{\rho} / \partial y>$ 0 et $\rho^{\prime}(t) \partial \bar{\rho} / \partial y<0$. D'autre part, dans une couche limite turbulente canonique, les paramètres intrinsèques comme le facteur de dissymétrie $S_{u^{\prime}}$ ou d'aplatissement $F_{u^{\prime}}$ sont liés directement aux variables internes. En effet, le facteur de dissymétrie est la signature moyenne des événements énergétiques : les balayages et les éjections. Il est, à cet égard, un indicateur indiquant la contribution statistique moyenne de chaque type d'événement. Dans la présente étude, les balayages sont plus marqués par $\rho^{\prime}(t)$ qu'ils le sont par $u^{\prime}(t)$ en vue de la correspondance qualitative entre le comportement dynamique de $\rho^{\prime}(t)$ et de la vitesse instantanée normale à la paroi $v^{\prime}(t)$. D'autre part, le facteur d'aplatissement est lié au caractère intermittent des fluctuations de vitesse et de densité. Plus la valeur de ce facteur est élevée, plus le signal est constitué de bouffées compactes isolées dans le temps.

\subsection{Principe de l'expérience}

Le principe de l'expérience consiste à injecter tangentiellement à la paroi dans une couche limite turbulente un gaz de densité différente de celle de l'écoulement externe à travers une fente d'épaisseur $e=3 \mathrm{~mm}$ formée entre deux plaques (Fig. 1). Un tel procédé permet d'engendrer une différence de densité importante. En effet, le développement du jet pariétal est schématiquement constitué de deux zones (Fig. 2) :

- Une première zone, où la couche limite développée sur la plaque supérieure rencontre l'écoulement de Poiseuille formé dans la fente d'injection. Ceci donne naissance à une couche de mélange se développant jusqu'à atteindre la plaque inférieure. Cette région est caractérisée par une très forte agitation due à d'importantes fluctuations de densité.

- Une deuxième zone pour un régime final où le profil de vitesse se rapproche de celui d'une couche limite turbulente standard, mais combiné à un gradient normal de densité. 


\begin{tabular}{ccccccccc}
\hline Profil $x(\mathrm{~mm})$ & 1 & 10 & 25 & 35 & 50 & 100 & 125 & 150 \\
\hline$x / e$ & 0,33 & 3,33 & 8,33 & 11,67 & 16,67 & 33,33 & 41,67 & 50 \\
\hline$x / \delta$ & 0,05 & 0,50 & 1,25 & 1,75 & 2,50 & 5,0 & 6,25 & 7,50 \\
\hline \multirow{2}{*}{$x^{+}\left(=x u_{\tau} / \nu\right)$} & 9 (air) & 87 & 209 & 304 & 448 & 986 & 1238 & 1492 \\
& $20(\mathrm{He})$ & 179 & 339 & 471 & 668 & 1182 & 1406 & 1663 \\
\hline \multirow{2}{*}{$\delta_{\rho} / \delta_{u}$} & $0,2($ air $)$ & 0,25 & 0,25 & 0,35 & 0,45 & 0,50 & 0,65 & 0,65 \\
& $0,2(\mathrm{He})$ & 0,25 & 0,40 & 0,45 & 0,50 & 0,70 & 0,80 & 0,85 \\
\hline
\end{tabular}

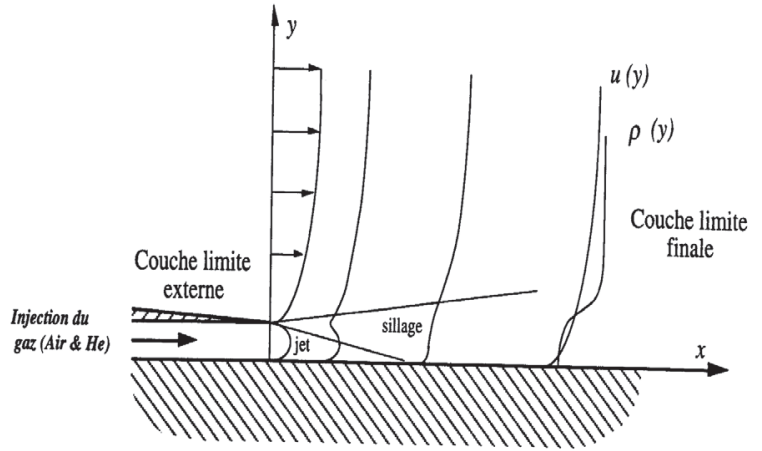

Fig. 2. Schématisation du développement du jet pariétal.

Les essais ont été effectués dans une soufflerie, où règne la pression atmosphérique, en injectant alternativement de l'air $\left(\rho_{\text {air }}=1,293 \mathrm{~kg} \cdot \mathrm{m}^{-3}\right)$ et de l'hélium $\left(\rho_{\mathrm{He}}=\right.$ $0,1785 \mathrm{~kg} \cdot \mathrm{m}^{-3}$ ) dans un mélange à densité constante. Les mesures ont été réalisées par une sonde d'interférence développée et expérimentée initialement par Way et Libby [20,21]. Elle est constituée de deux éléments sensibles (un fil et un film chauds) placés perpendiculairement l'un par rapport à l'autre ainsi que par rapport à l'écoulement. Son principe de fonctionnement repose sur l'influence thermique exercée par le film chaud sur le fil situé en amont. La faible distance entre les deux éléments sensibles $(\sim 25 \mu \mathrm{m})$ et la taille $\mathrm{du}$ film $\left(d_{\text {film }}=70 \mu \mathrm{m}\right)$ font que le fil se trouve dans le champ thermique du film. Dans nos travaux, la sonde d'interférence a été utilisée avec l'élément amont, chauffé plus que l'élément aval, car il a été montré [2] que cette configuration donnait lieu à un réseau d'étalonnage mieux conditionné qu'avec la disposition inverse des surchauffes utilisées par Way et Libby [20, 21].

\subsection{Conditions expérimentales}

Les résultats du présent travail sont obtenus dans les conditions expérimentales suivantes :

- vitesse de l'écoulement externe $U_{\infty}=5,8 \mathrm{~m} . \mathrm{s}^{-1}$,

- densité de l'écoulement externe $\rho_{\infty}=0,996 \mathrm{~kg} \cdot \mathrm{m}^{-3}$,

- vitesse d'injection des gaz (air \& hélium) $U_{d}=$ $2 \mathrm{~m} . \mathrm{s}^{-1}$

- rapports de densité : $\rho_{\mathrm{He}} / \rho_{\infty}=0,2$ et $\rho_{\text {air }} / \rho_{\infty}=1,3$.

\section{Résultats et discussion}

\subsection{Champs moyens et fluctuants}

Les mesures menées par Harion [2], des champs moyens de vitesse et de densité ainsi que le frottement pariétal, ont montré que le développement du jet pariétal est régi principalement par le rapport entre la vitesse débitante d'injection du gaz dans la fente et celle de l'écoulement externe $\left(U_{\mathrm{d}} / U_{\infty}=0,35\right)$. De plus, le développement du jet pariétal est plus rapide dans le cas d'une injection d'hélium que dans le cas d'une injection d'air. Ceci s'explique : pour une injection d'hélium, la région de transition est relativement plus courte $(x / e \approx$ 17) que celle correspondant à une injection d'air $(x / e \approx$ 25 ), ce qui provoque une chute importante du frottement pariétal moyen dans cette région. Ceci est essentiellement lié à la différence de densité et donc de la viscosité cinématique à la paroi. En effet la fraction molaire d'air à la paroi vaut environ 0,64 pour l'hélium et 0,85 pour l'air. Entre ces deux valeurs, la viscosité dynamique varie très peu $(\Delta \mu \approx 3 \%)$.

On résume dans le tableau ci-dessus les abscisses des sections, en aval de la fente d'injection des gaz, où les mesures sont effectuées.

L'épaisseur de la couche limite $\left(\delta_{u}: \bar{U}=0,99 U_{\infty}\right)$, dans une section de mesure donnée, est à peu près constante pour les deux gaz injectés. Les profils moyens de vitesse ne sont que très peu affectés par la nature du gaz injecté. Cependant, l'injection d'hélium donne lieu à des vitesses moyennes légèrement supérieures près de la paroi $(y / \delta<0,4)$, dans la région du développement de la couche limite $(x / e>20)$. Ceci conduit à une vitesse de frottement $u_{\tau}$, déterminée à partir de la loi universelle de la couche limite turbulente, plus élevée de $10 \%$ pour une injection d'hélium. Indépendamment du gaz injecté, l'échelle interne de la vitesse $u_{\tau}$ de la couche limite formée dans la zone du régime développé varie dans la direction principale de l'écoulement. Cette variation est essentiellement liée au rapport de densité $\overline{\rho_{\mathrm{p}}} / \rho_{\infty}$ ( $\rho_{\mathrm{p}}$ est la densité à la paroi) agissant sur la viscosité à la paroi $\nu_{\mathrm{p}}$. Cependant, Cheng et $\mathrm{Ng}[1,22]$ n'ont pas noté ce changement de $u_{\tau}$ pour une couche limite fortement chauffée. Ils ne mentionnent qu'une faible variation, d'environ $2 \%$, de la valeur de $u_{\tau}$ dans le cas d'une paroi chauffée $\left(T_{\mathrm{p}}=1100 \mathrm{~K}\right)$ par rapport au cas isotherme. Ce résultat est surprenant si l'on considère les propriétés connues de l'air où la viscosité cinématique présente une augmentation d'un facteur 9 entre $293 \mathrm{~K}$ 
et $1100 \mathrm{~K}$ (Bejan [23]). Les résultats obtenus affichent une légère différence par rapport à la loi universelle (cas d'une couche limite turbulente sur une plaque plane classique) et aux mesures de Purtell et al. [24] concernant des écoulements de couche limite à bas nombre de Reynolds. Ceci explique que les profils moyens de vitesse n'étant que peu affectés par la différence de densité.

Les mesures de densité mettent en évidence une dilution très rapide et de très forts gradients de densité dans la zone initiale de l'écoulement. Dans la zone du régime développé, ces gradients atteignent un comportement asymptotique, pour lequel le rapport de densité $\overline{\rho_{\mathrm{p}}} / \rho_{\infty}$ évolue lentement avec $x$ et tend vers une valeur de l'ordre de 0,8 à la fin de la section d'essai, pour une injection d'hélium. Les mesures des fluctuations de vitesse et de densité montrent que, dans la région proche de la paroi, l'intensité turbulente est renforcée par l'injection l'hélium. En effet, Cheng et $\mathrm{Ng}$ [1] trouvent la même tendance dans une couche limite fortement chauffée, où $\sqrt{\overline{u^{\prime 2}}} / U_{\infty} \approx 12 \%$ à $y / \delta=0,05$ lorsque $T_{\mathrm{p}}=1100 \mathrm{~K}$ alors que ces fluctuations n'atteignent que $10 \%$ dans le cas isotherme. Le mélange entre les deux écoulements, initialement à densités différentes, donne naissance à des fluctuations très importantes au niveau de la couche de mélange. Le niveau des fluctuations de densité, au voisinage de la paroi, est plus élevé lorsque le gaz injecté est de l'hélium dans la zone du développement du jet pariétal. Dans la région proche de l'injection, ces fluctuations sont très importantes $\left(\sqrt{\overline{\rho^{\prime 2}}} / \rho_{\infty} \approx 20 \%\right)$. Cependant, Cheng et $\mathrm{Ng}$ [1] montrent que les fluctuations de densité atteignent un maximum de $\sqrt{\overline{\rho^{\prime 2}}} / \rho_{\infty} \approx 12 \%$. Ceci peut être dû aux différences dans les conditions expérimentales (un chauffage continu de la paroi dans l'étude de Cheng et $\mathrm{Ng}$ [1], et une discontinuité due à l'injection du gaz à densité différente dans la présente étude). Les mesures à la paroi confirment les résultats déduits des mesures de vitesse et de densité. Il est montré que le frottement pariétal en régime développé n'est pas affecté par la nature du gaz injecté. Cependant la vitesse de frottement $u_{\tau}$ est affectée de façon significative par l'injection de gaz de densités différentes. De plus, les fluctuations du frottement pariétal, déterminés à l'aide d'un film chaud collé sur la paroi, ne subissent pas une influence notable de la nature du gaz injecté. Dangel et al. [25], par une technique semblable, trouvent un résultat du même ordre de grandeur $\left(\sqrt{\overline{\tau_{\mathrm{p}^{2}}^{\prime}}} / \overline{\tau_{\mathrm{p}}}=13 \%\right)$, dans un écoulement turbulent homogène. Harion [2] montre que ces fluctuations sont de l'ordre de $\sqrt{\overline{\tau_{\mathrm{p}^{2}}^{\prime}}} / \overline{\tau_{\mathrm{p}}}=15 \%$, dans la zone de l'écoulement développé. Ces valeurs restent, cependant, inférieures à la valeur généralement admise de $\sqrt{\overline{\tau_{\mathrm{p}^{2}}^{\prime}}} / \overline{\tau_{\mathrm{p}}}=36 \%$ en écoulement homogène. D'autre part, l'injection d'hélium donne à la paroi des niveaux importants de fluctuations de densité, dans la zone de transition de l'ordre de $\sqrt{\overline{\rho_{\mathrm{p}^{2}}^{\prime}}} / \rho_{\infty} \approx 10 \%$ à $x / e=10$. Ces fluctuations restent supérieures à celles données par l'injection d'air dans les deux régions du développement du jet pariétal. Ce résultat peut s'expliquer, en partie, par l'influence de la nature du gaz injecté sur la vitesse de frottement $u_{\tau}=\sqrt{\overline{\tau_{\mathrm{p}}} / \overline{\rho_{\mathrm{p}}}}$. L'analyse de ces résultats permet une étude détaillée des structures présentes dans ce type de couche limite.

Dans les mêmes conditions expérimentales et ayant pour objectif de connaître le détail des mécanismes de la couche limite turbulente en présence de variations importantes de densité, il est important d'analyser la distribution instantanée des fluctuations de densité et de vitesse. Cette distribution nous donne une information sur la corrélation vitesse-densité. L'étude de cette corrélation double permet de mettre en évidence deux modes dominants des fluctuations de vitesse et de densité dans le mouvement du fluide (éjection-balayage). Cette corrélation est, aussi, une variable importante pour comprendre l'effet des fortes différences de densité sur le comportement des structures de la turbulence et leurs contributions aux mouvements du fluide dans la couche limite turbulente étudiée.

\subsection{Statistiques des moments d'ordre élevé}

\subsubsection{Facteurs de dissymétrie}

Les figures 3 et 4 montrent les profils des facteurs de dissymétrie $S_{u^{\prime}}=\overline{u^{\prime 3}} /{\sqrt{u^{\prime 2}}}^{3}$ et $S_{\rho^{\prime}}={\overline{\rho^{\prime 3}}}^{\prime}{\sqrt{\rho^{\prime 2}}}^{3}$ à différentes sections de mesure $x / \delta$. Les signes + et - dans $S_{\rho^{\prime}}$ se réfèrent respectivement à $\partial \bar{\rho} / \partial y<0$ (cas de l'injection d'air) et à $\partial \bar{\rho} / \partial y>0$ (cas de l'injection d'hélium).

Nous avons opté pour cette représentation, car elle permet de prendre en compte la distribution temporelle de $\rho^{\prime}(t)$, qui pour $u^{\prime}(t)<0$ ou $u^{t}(t)>0$ donnée, résulte respectivement en $\rho^{\prime}(t) \partial \bar{\rho} / \partial y>0$ et $\rho^{\prime}(t) \partial \bar{\rho} / \partial y<0$. D'autre part, dans une couche limite turbulente canonique, les paramètres intrinsèques comme le facteur de dissymétrie $S_{u^{\prime}}$ ou d'aplatissement $F_{u^{\prime}}$ sont liés aux variables internes. C'est la raison pour laquelle les distributions $S_{u^{\prime}}$ et $S_{\rho^{\prime}}$ sur les figures 3 et 4 sont données en fonction de $y^{+}=y / l_{\nu}$ où $l_{\nu}=u_{\tau \text { local }} / \nu_{\text {local }}$ est l'échelle interne locale. La relaxation du facteur de dissymétrie de $u^{\prime}$ s'effectue plus rapidement dans la couche externe que près de la paroi, aussi bien pour l'injection de l'air que pour l'injection d'hélium (Fig. 3). Ceci peut paraître curieux, de prime abord, car la couche interne a, en principe, une mémoire courte, alors que la couche externe a un temps de relaxation relativement long. N'oublions pas cependant que l'intervention dans la couche limite se fait à la paroi, et c'est principalement la région proche de la paroi qui est affectée par les changements structurels de la turbulence. $S_{u^{\prime}}$ croît près de la fente d'injection d'une façon appréciable et cette augmentation est nettement plus accentuée pour l'injection de l'air que l'injection d'hélium. La croissance du facteur de dissymétrie près d'une fente d'injection a été observée dans un contexte différent et peut s'expliquer par l'augmentation du flux convectif longitudinal de l'énergie turbulente 

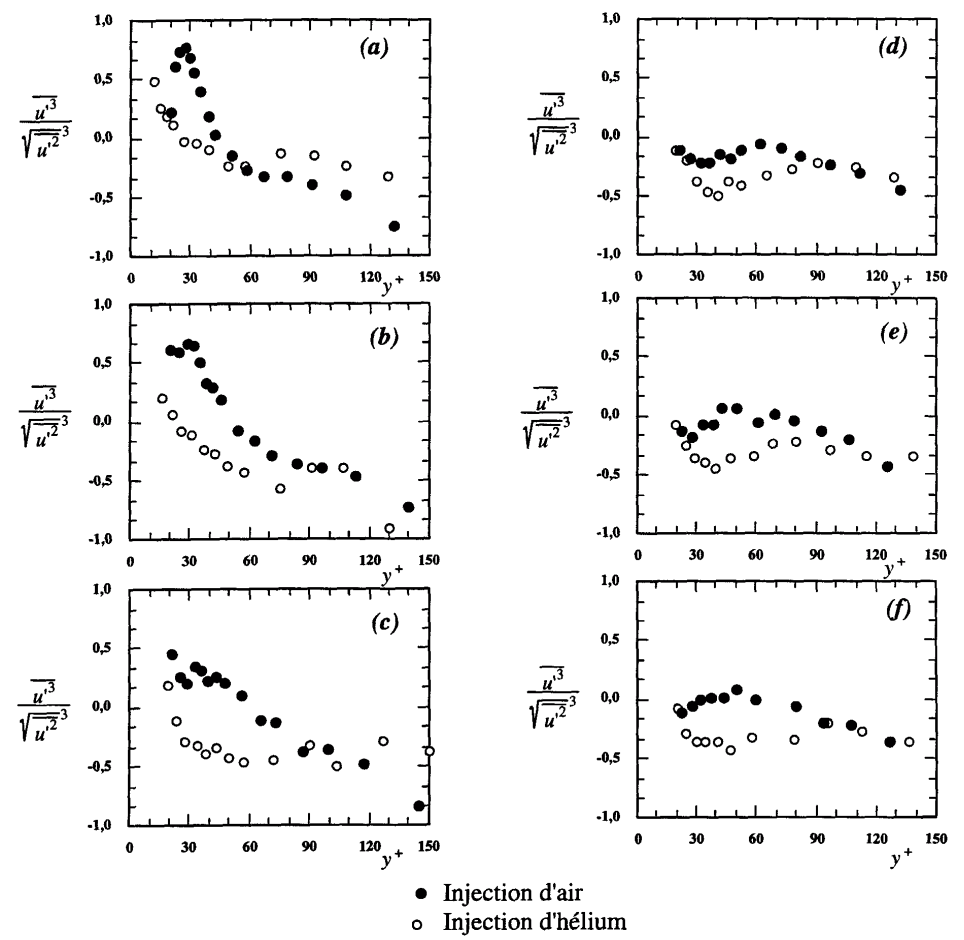

\begin{tabular}{|c|c|c|c|c|c|c|}
\hline Profil & $a$ & $b$ & $c$ & $d$ & $e$ & $f$ \\
\hline$x / e$ & 8,33 & 11,67 & 16,67 & 33,33 & 41,67 & 50 \\
\hline$x / \delta$ & 1,25 & 1,75 & 2,50 & 5,0 & 6,25 & 7,50 \\
\hline$\Delta \rho / \rho_{\infty}=\left(\overline{\rho_{\mathrm{p}}}-\rho_{\infty}\right) / \rho_{\infty}$ & $\begin{array}{l}0,15 \text { (Air) } \\
0,60(\mathrm{He}) \\
\end{array}$ & $\begin{array}{l}0,12 \\
0,45 \\
\end{array}$ & $\begin{array}{l}0,10 \\
0,37\end{array}$ & $\begin{array}{l}0,06 \\
0,20 \\
\end{array}$ & $\begin{array}{l}0,050 \\
0,200 \\
\end{array}$ & $\begin{array}{l}0,050 \\
0,200 \\
\end{array}$ \\
\hline$\left(-\left(\delta / \rho_{\infty}\right)(\partial \bar{\rho} / \partial y)\right)_{\mathrm{air}}$ & 0,80 & 0,70 & 0,50 & 0,18 & 0,13 & 0,13 \\
\hline$\left(\left(\delta / \rho_{\infty}\right)(\partial \bar{\rho} / \partial y)\right)_{\mathrm{He}}$ & 3,7 & 2,2 & 1,2 & 0,4 & 0,3 & 0,3 \\
\hline
\end{tabular}

Fig. 3. Profils du facteur de dissymétrie $S_{u^{\prime}}={\overline{u^{\prime 3}}} /{\sqrt{u^{\prime 2}}}^{3}$.

dans cette zone. Afin d'être plus clair, considérons le terme $-1 / 2 \partial\left(\overline{\rho u_{i}^{\prime} u_{i}^{\prime} u_{j}^{\prime}}\right) / \partial x_{j}$ de la diffusion turbulente de l'équation de l'énergie cinétique : $k=1 / 2 \overline{u_{i}^{\prime} u_{i}^{\prime}}$, i.e. $\partial\left(\overline{\rho k u_{j}}\right) / \partial x_{j}$. Étant donné près de la fente d'injection $\partial / \partial x_{1}$ ne peut pas être négligeable, on trouve le terme $-1 / 2 \partial\left(\overline{\rho u^{\prime 3}}\right) / \partial x_{1}=-1 / 2 \partial\left(\bar{\rho} \overline{u^{\prime 3}}\right) / \partial x_{1}-1 / 2 \partial\left(\overline{\rho^{\prime} u^{\prime 3}}\right) / \partial x_{1}$. L'intégration de $\partial\left(\bar{\rho} \bar{k} \overline{u_{j}}\right) / \partial x_{j}$ entre deux plans $x / \delta=0$ et $x / \delta$ où le facteur de dissymétrie $S_{u^{\prime}}$ devient relativement faible, indique alors qu'il y a un flux net longitudinal de l'ordre de $\left[\bar{\rho} \overline{u^{\prime 3}}\right]_{x / \delta \sim 0}$ qui représente un transfert net des régions d'intensité turbulente forte vers les zones d'intensité faible. Bien que cet argument permette d'expliquer l'augmentation du facteur de dissymétrie $S_{u^{\prime}}$ dans la zone du développement du jet pariétal, il est difficile de comprendre pourquoi le transport d'énergie latérale est plus important pour $\Delta \rho=\rho_{\text {inj }}-\rho_{\infty}>0$ i.e. pour l'injection de l'air, car il est clairement établi à partir de la figure 3 que $S_{u^{\prime} \Delta \rho>0}$ est systématiquement plus large que $S_{u^{\prime} \Delta \rho<0}$. Une explication initiale possible est que la relaxation est plus rapide lorsque le gradient $\partial \bar{\rho} / \partial y$ est plus fort, ce qui est le cas pour l'injection d'hélium.
La relaxation n'est pas terminée lorsque les gradients $\partial / \partial x$ deviennent faibles, i.e. lorsque $x / \delta \geq 5$ (Figs. $3 \mathrm{~d}-\mathrm{f}$ ). La couche limite est essentiellement sous l'influence de $\partial \bar{\rho} / \partial y$, et de $\Delta \rho / \rho_{\infty}=\left(\bar{\rho}_{\mathrm{p}}-\rho_{\infty}\right) / \rho_{\infty}$, où $\bar{\rho}_{\mathrm{p}}$ est la masse volumique à la paroi. On peut préciser le rôle de $\partial \bar{\rho} / \partial y$ dans la modification de la structure de turbulence. Dans la zone établie près de la paroi, il paraît logique de négliger les terme advectifs de l'équation d'énergie et de ne retenir au premier ordre, que les variations suivant $y$. Ceci résulte en :

$$
\begin{aligned}
\overline{\rho u^{\prime} v^{\prime}} \frac{\partial \bar{U}}{\partial y} & +\frac{\partial}{\partial y} \overline{\rho v^{\prime} \frac{u_{i}^{\prime} u_{i}^{\prime}}{2}} \\
& +\frac{\partial}{\partial y}\left(\overline{p^{\prime} v^{\prime}}\right) \sim \frac{\partial}{\partial y} \overline{\left[\mu u_{i}^{\prime}\left(\frac{\partial v^{\prime}}{\partial x_{i}}+\frac{\partial u^{\prime}}{\partial y}\right)\right]}-\bar{\rho} \varepsilon
\end{aligned}
$$

Si on ne considère que l'effet du gradient de masse volumique moyenne, on peut écrire :

$$
\overline{\rho u^{\prime} v^{\prime}} \sim \bar{\rho} \overline{u^{\prime} v^{\prime}} \quad \text { et } \overline{\rho v^{\prime} \frac{u_{i}^{\prime} u_{i}^{\prime}}{2}} \sim \bar{\rho} \overline{v^{\prime} \frac{u_{i}^{\prime} u_{i}^{\prime}}{2}}
$$



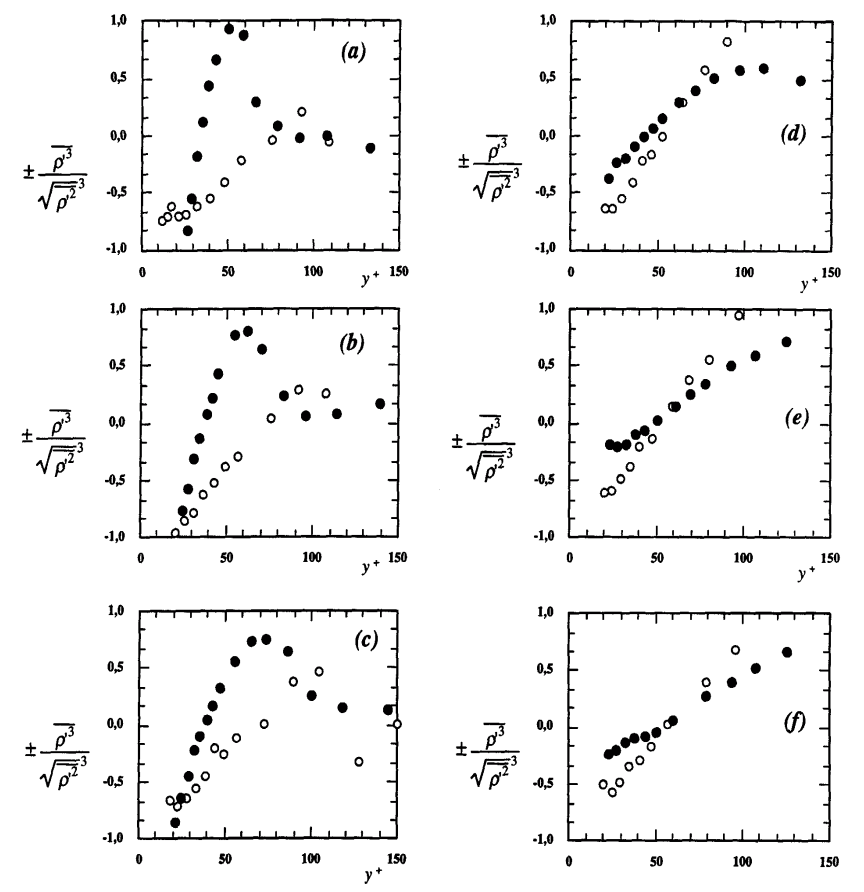

- Injection d'air

- Injection d'hélium

\begin{tabular}{|c|c|c|c|c|c|c|}
\hline Profil & $\boldsymbol{a}$ & $\boldsymbol{b}$ & $\boldsymbol{c}$ & $\boldsymbol{d}$ & $\boldsymbol{e}$ & $\boldsymbol{f}$ \\
\hline$x / e$ & 8,33 & 11,67 & 16,67 & 33,33 & 41,67 & 50 \\
\hline$x / \delta$ & 1,25 & 1,75 & 2,50 & 5,0 & 6,25 & 7,50 \\
\hline$\Delta \rho / \rho_{\infty}=\left(\overline{\rho_{\mathrm{p}}}-\rho_{\infty}\right) / \rho_{\infty}$ & $0,15($ Air $)$ & 0,12 & 0,10 & 0,06 & 0,050 & 0,050 \\
& $0,60(\mathrm{He})$ & 0,45 & 0,37 & 0,20 & 0,200 & 0,200 \\
\hline$\left(-\left(\delta / \rho_{\infty}\right)(\partial \bar{\rho} / \partial y)\right)_{\mathrm{air}}$ & 0,80 & 0,70 & 0,50 & 0,18 & 0,13 & 0,13 \\
\hline$\left(\left(\delta / \rho_{\infty}\right)(\partial \bar{\rho} / \partial y)\right)_{\mathrm{He}}$ & 3,7 & 2,2 & 1,2 & 0,4 & 0,3 & 0,3 \\
\hline
\end{tabular}

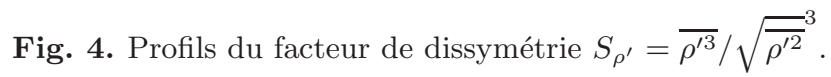

de sorte que

$$
\begin{aligned}
\overline{u^{\prime} v^{\prime}} \frac{\partial \bar{U}}{\partial y}+\frac{\partial}{\partial y} \overline{v^{\prime}} \frac{u_{i}^{\prime} u_{i}^{\prime}}{2}+\frac{1}{\bar{\rho}} \frac{\partial \bar{\rho}}{\partial y} \overline{v^{\prime} \frac{u_{i}^{\prime} u_{i}^{\prime}}{2}} \\
+\frac{1}{\bar{\rho}} \frac{\partial}{\partial y}\left(\overline{p^{\prime} v^{\prime}}\right) \sim \frac{1}{\bar{\rho}} \frac{\partial}{\partial y} \mu \overline{u_{i}^{\prime}\left[\frac{\partial v^{\prime}}{\partial x_{i}}+\frac{\partial u^{\prime}}{\partial y}\right]}-\varepsilon
\end{aligned}
$$

La comparaison de cette équation avec celle correspondant à la couche limite avec $\Delta \rho=0$ :

$$
\begin{aligned}
\overline{u^{\prime} v^{\prime}} \frac{\partial \bar{U}}{\partial y}+\frac{\partial}{\partial y} \overline{v^{\prime}} & \frac{u_{i}^{\prime} u_{i}^{\prime}}{2} \\
& +\frac{\partial}{\partial y} \overline{\left(v^{\prime} \frac{p^{\prime}}{\rho}\right)} \sim \frac{\mu}{\bar{\rho}} \frac{\partial}{\partial y} \overline{u_{i}^{\prime}\left[\frac{\partial v^{\prime}}{\partial x_{i}}+\frac{\partial u^{\prime}}{\partial y}\right]}-\varepsilon
\end{aligned}
$$

montre que l'une des différences essentielles provient du terme $\frac{1}{\overline{\bar{\rho}}} \overline{v^{\prime} \frac{u_{i}^{\prime} u_{i}^{\prime}}{2}} \frac{\partial \bar{\rho}}{\partial y}$ que l'on peut interpréter comme une contribution du gradient de densité à la convection turbulente. Dans une couche limite canonique le terme
$(1 / 2 \rho) \overline{v^{\prime 3}}$ de l'équation correspondante est de l'ordre de 0,3 en unité pariétale dans la couche à $y^{+} \geq 30$ ce qui s'explique par la prédominance des éjections avec $v^{\prime} \geq 0$ dans cette zone. Si ce phénomène n'est pas beaucoup affecté par la présence de $\partial \bar{\rho} / \partial y$, on peut alors estimer le terme $A=\frac{1}{\bar{\rho}} \overline{v^{\prime} \frac{u_{i}^{\prime} u_{i}^{\prime}}{2}} \frac{\partial \bar{\rho}}{\partial y}$ pour l'injection d'hélium à $\frac{1}{\rho} S_{v^{\prime}}\left(\overline{v^{\prime 2}}\right)^{3 / 2} \frac{\mathrm{d} \bar{\rho}}{\mathrm{d} y}$ (en ne tenant pas compte du facteur deux et ne considère que le terme en $v^{\prime 3}$ ). Les résultats de la couche limite canonique (Kim et al. [26]) fournissent $S_{v^{\prime}} \sim 0,3$ et $v^{\prime+} \sim 1$ pour $y^{+} \geq 50$. On a alors $A \sim \frac{0,3}{\rho} \frac{\mathrm{d} \bar{\rho}}{\mathrm{d} y} u_{\tau}^{3}$. On compare ce terme au terme prépondérant de la production d'énergie turbulente : $B=\overline{u^{\prime} v^{\prime}} \frac{\partial \bar{U}}{\partial y}$. L'étude de Kim [14] fournit : $\overline{u^{\prime} v^{\prime}} \sim 0,7 u_{\tau}^{3}$. D'où $B \sim 0,7 u_{\tau}^{3} \frac{\partial u^{+}}{\partial y}$. De plus, $\mathrm{d} u^{+} \sim 2,5 \mathrm{~d} y^{+} / y^{+}$, d'où $\frac{A}{B} \sim 10 \frac{1}{\rho} \frac{\partial \bar{\rho}}{\partial y^{+}}$.

Près de la fente d'injection, on trouve $A / B \sim 0,3$ alors que pour $x / \delta=5, A / B \sim 0,02$. On voit que l'effet du gradient de la masse volumique moyenne peut intervenir au début de la couche limite de concentration, 

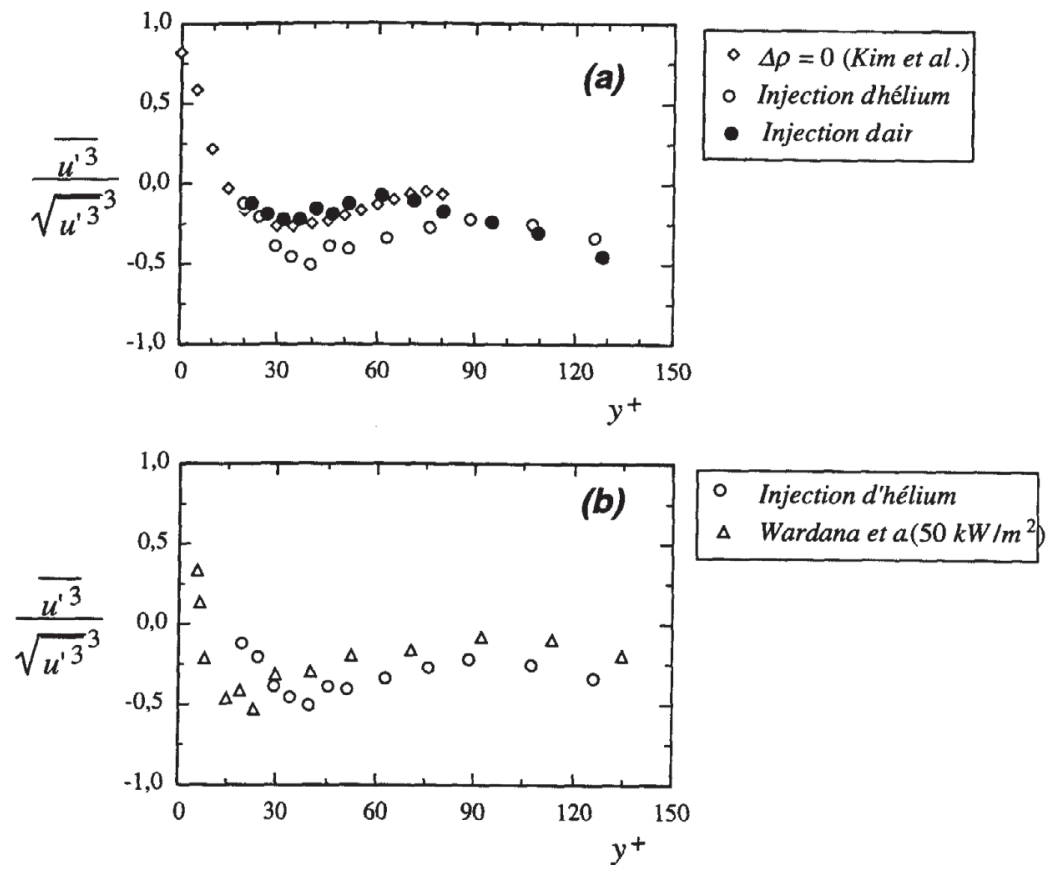

Fig. 5. Comparaison des facteurs de dissymétrie $S_{u^{\prime}}={\overline{u^{\prime 3}}}^{y^{+}}{\sqrt{u^{\prime 2}}}^{3}$ (pour la présente étude, $x / \delta=5$ ).

mais qu'il devrait être très atténué à $x / \delta=5$. C'est donc bien un effet de lente relaxation qui peut expliquer la persistance de valeurs fortement négatives du facteur de dissymétrie $S_{u^{\prime}}$ correspondant à l'injection d'hélium dans la zone développée (Figs. 3d-f). Dans la région initiale, le gradient de densité moyenne est nettement plus important pour une injection d'hélium que pour une injection d'air. On peut s'attendre à ce que l'effet sur la production $\overline{u^{\prime} v^{\prime}} \partial \bar{U} / \partial y$ soit nettement plus important dans ce cas d'injection. Ceci peut s'expliquer par les différences persistantes obtenues à $x / \delta \geq 5$ entre les deux profils

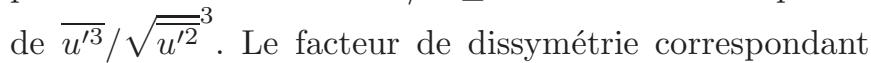
à l'injection d'hélium est systématiquement fortement négatif dans toute la couche limite interne dans la zone développée comme en témoignent les figures 3d-f. Étant donné que la couche interne est majoritairement sous l'influence des événements du type éjection $\left(u^{\prime}<0, v^{\prime}>0\right)$, les valeurs systématiquement négatives de $\left(\overline{u^{\prime 3}} / \sqrt{{\overline{u^{\prime 2}}}^{3}}\right)_{\mathrm{He}}$ signifient un renforcement de ce type d'événement associé à un gradient $\partial \bar{\rho} / \partial y>0$ fort. Cet effet montre que la densité ne peut plus être considérée comme un scalaire passif, lorsque $\partial \bar{\rho} / \partial y$ dépasse un certain seuil, et qu'il y a alors un effet rétroactif de $\Delta \rho$ sur la structure fine de la turbulence. Il est utile à cette étape de l'analyse de confronter les distributions du facteur de dissymétrie avec $S_{u^{\prime}}$ de la couche limite standard. La figure 14a montre les distributions de $\left({\overline{u^{\prime 3}}}^{2} \sqrt{{\overline{u^{\prime 2}}}^{3}}\right)_{\mathrm{He}}$ et de $\left({\overline{u^{\prime 3}}} / \sqrt{{\overline{u^{\prime 2}}}^{3}}\right)_{\mathrm{Air}}$ à $x / \delta=5$ en comparaison avec $S_{u^{\prime}}$ obtenu par la simulation directe de Kim et al. [26] lorsque $\Delta \rho=0$. On remarque une parfaite correspondance des profils du facteur de dissymétrie de $u^{\prime}$ pour l'injection d'air et celui

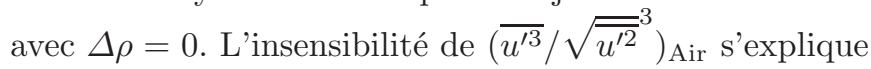

par les faibles valeurs de $(\partial \bar{\rho} / \partial y)_{\text {Air }}$ qu'on peut affecter à la redistribution des événements énergétiques dans les quadrants. Pour ce cas d'injection, on peut remarquer (tableau de la Fig. 3) que le gradient de $\bar{\rho}$ dans la première section de mesure présentée est à peu près 5 fois plus faible que pour l'injection d'hélium. En revanche, $\left(\overline{u^{\prime 3}} / \sqrt{{\overline{u^{\prime 2}}}^{3}}\right)_{\mathrm{He}}$ est significativement plus important dans la couche tampon et au début de la couche limite logarithmique, ce qu'on peut attribuer aux gradients relativement plus forts de $(\partial \bar{\rho} / \partial y)_{\mathrm{He}}$ près de l'injection, qui intensifient les événements avec $u^{\prime}(t)<0$. Nous comparons sur la figure $5 \mathrm{~b}$ le facteur de dissymétrie de $u^{\prime}$ avec $\partial \bar{\rho} / \partial y>0$ (injection de l'hélium) avec celui obtenu par Wardana et al. [11] pour un écoulement sur une paroi fortement chauffée et pour un flux de chaleur $q=50 \mathrm{~kW} \cdot \mathrm{m}^{-2}$. Les deux profils coïncident qualitativement, ce qui montre encore une fois la similitude entre les deux écoulements. Wardana et al. [11] ont maintenu la température à la paroi à $1000 \mathrm{~K}$ ce qui correspond approximativement à $\overline{\rho_{\mathrm{p}}} / \rho_{\infty} \sim 1 / 3$ et à peu de chose près à l'injection d'hélium dans nos expériences. Les différences fondamentales entre notre étude et celle de Wardana et al. [11] proviennent $\mathrm{du}$ fait que ces derniers traitent un écoulement pleinement développé en canal, alors qu'il y a des effets profonds de relaxation dans les expériences que nous avons menées. On ne peut en effet s'attendre qu'à une concordance qualitative, car les profils des gradients $\partial \bar{\rho} / \partial x_{i}$ sont significativement différents dans les deux études. Dans un écoulement turbulent développé sur une paroi fortement chauffée, le gradient $\partial \bar{\rho} / \partial x \sim 0$. En outre le gradient normal $\partial \bar{\rho} / \partial y$ est lié au gradient de température $\partial \bar{T} / \partial y$, qui varie comme $\partial T^{+} / \partial y^{+}=1$ dans la souscouche visqueuse et qui diminue dans la couche logarithmique $y^{+} \geq 50$ suivant $\partial T^{+} / \partial y^{+} \sim A / \partial y^{+}$où $A$ est la 
constante de Van Karman. Dans l'étude présentée ici, en revanche, $\partial \bar{\rho} / \partial x$ ne peut être négligeable dans la région initiale et $\partial \bar{\rho} / \partial y$ est presque constant à travers toute la couche interne dans la zone développée de l'écoulement, alors qu'à la paroi la condition d'imperméabilité impose $(\partial \bar{\rho} / \partial y)_{y=0}=0$. Des différences qualitatives profondes observées entre les deux études peuvent par conséquent être liées à un forçage différent de $\partial \bar{\rho} / \partial y$. La figure 6 qui compare le profil de $\partial\left(\bar{\rho} / \rho_{\infty}\right) / \partial y^{+}$obtenu dans la zone développée $(x / \delta=5)$, avec celui obtenu sur une paroi chauffée [20], illustre la discussion ci-dessus. La re-

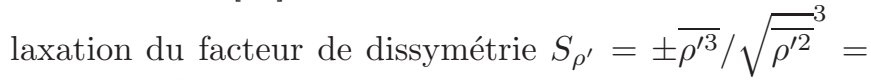

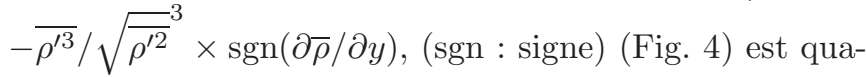
litativement similaire à celle de $S_{u^{\prime}}$ (Fig. 3), avec cependant certaines différences importantes. Il faut noter en premier lieu que les profils de $S_{\rho^{\prime}}$ correspondant à l'injection d'hélium et d'air s'écartent beaucoup plus significativement dans la première zone de développement du jet pariétal, i.e. pour $x / \delta<2,5$; comparés avec $S_{u^{\prime}}$ dans la même région. La relaxation de $S_{\rho^{\prime}}$ a lieu cependant approximativement à la même distance $x / \delta \sim 5$ comme en témoignant les figures $4 \mathrm{~d}-\mathrm{f}$. L'effet du gradient $\partial \bar{\rho} / \partial x$ se fait sentir par conséquent de la même façon sur les distributions de $S_{u^{\prime}}$ et de $S_{\rho^{\prime}}$. L'étude détaillée de la distribution de $S_{\rho^{\prime}}$ dans la zone de l'écoulement développé paraît contradictoire au premier abord, au vu de la discussion menée concernant $S_{u^{\prime}}$. En effet, on remarque sur la figures $4 \mathrm{~d}-\mathrm{f}$, que pour $y^{+} \leq 50, S_{\rho^{\prime}}$ est significativement négatif, ce qui impliquerait une intensification des balayages. Or le comportement des profils de $S_{u^{\prime}}$ indiquent au contraire une intensification des éjections comme nous l'avons discuté auparavant. Il faut noter également que $S_{\rho^{\prime}}$ est négatif pour $\partial \bar{\rho} / \partial y>0$ (injection d'hélium), presque dans toute la couche limite interne dans la zone du développement $(x / \delta<5)$, indiquant la prédominance de la signature des balayages. La discordance entre $S_{u^{\prime}}<0$ (éjection) et $S_{\rho^{\prime}}<0$ (balayage) pour $y^{+}<50$ s'explique justement par le fait que le facteur de dissymétrie n'est que la signature moyenne des événements énergétiques et qu'il n'est, à cet égard, qu'un indicateur indiquant la contribution statistique moyenne de chaque type d'événement. Ce n'est pas impossible pour l'instant que les balayages soient plus marqués par $\rho^{\prime}(t)$ qu'ils le sont pour $u^{\prime}(t)$. La même contradiction existe quand on regarde de près les profils de $S_{u^{\prime}}$ et $S_{\theta^{\prime}}$ de Wardana et al. [10]. Nous confrontons sur la figure 7 a la distribution de $S_{\theta^{\prime}}$ obtenue par ces auteurs avec celle de $S_{\rho^{\prime}}$ pour l'injection d'hélium. On remarque au premier abord une concordance qualitative satisfaisante entre les deux séries de mesure. En second lieu, on note que $S_{\theta^{\prime}}<0$ pour $y^{+} \leq 30$, ce qui indique la prédominance des balayages, alors que $S_{u^{\prime}}$ est également négatif dans cette zone (voir Fig. 5b). La zone dans laquelle $S_{\rho^{\prime}}<0$ est cependant significativement plus large dans le cas de l'injection d'hélium, comparée à celle de $S_{\theta^{\prime}}$ sur une paroi fortement chauffée.

Les statistiques des fluctuations de température doivent en réalité être confrontées à celles de la

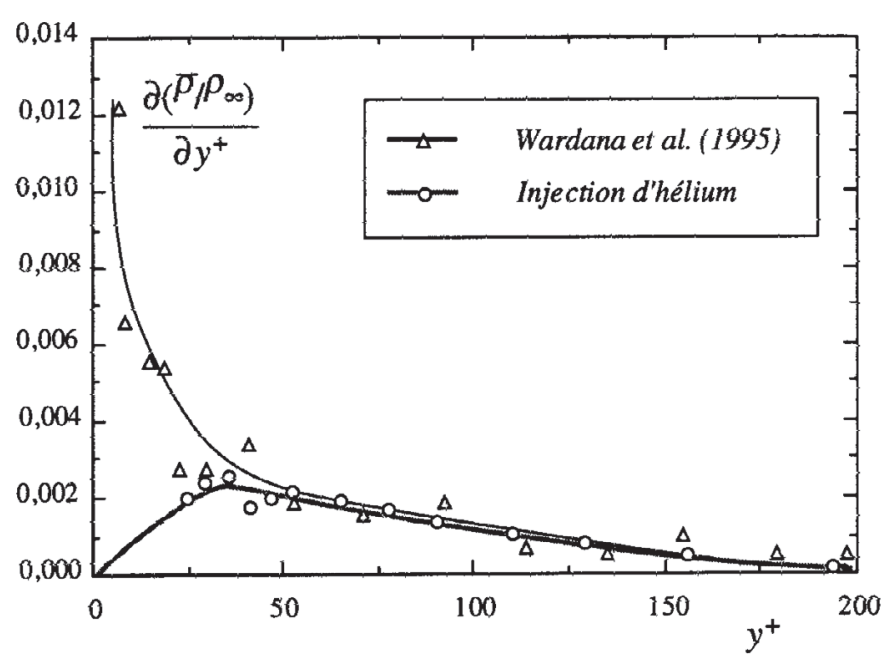

Fig. 6. Profils du gradient normal de densité (pour la présente étude, $x / \delta=5)$.

concentration plutôt que celles de la masse volumique. L'équation qui gère la variance d'un scalaire, comme la température ou la concentration, est en effet fondamentalement différente de celle qui gouverne la variance de la masse volumique. Pour le scalaire $c^{\prime}$ (concentration), on a en effet :

$$
\begin{aligned}
& \frac{\partial}{\partial x_{k}}\left(\overline{\rho c^{\prime 2} U_{k}}\right)= \\
& -2 \overline{\rho c^{\prime} u_{k}^{\prime}} \frac{\partial \bar{C}}{\partial x_{k}}+\frac{\partial}{\partial x_{k}}\left(-2 \overline{c^{\prime} q_{k}^{\prime}}-\overline{\rho c^{\prime 2} u_{k}^{\prime}}\right)-2 \varepsilon_{f}-2 \overline{\rho c^{\prime}} \frac{\partial \bar{U}}{\partial x_{k}} \frac{\partial \bar{C}}{\partial x_{k}}
\end{aligned}
$$

où : $q_{k}^{\prime}=-\alpha \frac{\partial c^{\prime}}{\partial x_{k}}$, représente le flux fluctuant du scalaire par diffusion moléculaire, et $\varepsilon_{f}$ est la pseudo-dissipation. Cette équation est déduite de celle de la concentration instantanée $C(t)$ i.e.

$$
\rho \frac{D C}{D t}=\frac{\partial}{\partial x_{k}}\left(\alpha \frac{\partial C}{\partial x_{k}}\right)
$$

La variance de la masse volumique, en revanche, est :

$\frac{\partial}{\partial x_{k}}\left(\overline{\rho^{\prime 2} U_{k}}\right)=-2 \overline{\rho u_{k}^{\prime}} \frac{\partial \bar{\rho}}{\partial x_{k}}-\frac{\partial \overline{\rho^{\prime 2} u_{k}^{\prime}}}{\partial x_{k}}-\overline{\rho^{\prime 2} \frac{\partial U_{k}}{\partial x_{k}}}-2 \bar{\rho} \rho^{\prime} \frac{\partial u_{k}^{\prime}}{\partial x_{k}}$

qui est substantiellement différente. La figure $7 \mathrm{~b}$ compare $S_{\theta^{\prime}}$ de Wardana et al. $[20,21]$ avec $S_{c^{\prime}}$ obtenu dans la présente étude. Dans la couche visqueuse $y^{+} \leq 50$, les différences entre les deux distributions sont étonnamment moindres que celles de la couche externe. En tout état de cause, les positions $y^{+}$pour lesquelles $S_{\theta^{\prime}}$ et $S_{c^{\prime}}=$ changent de signe coïncident relativement bien comparé avec celles de $S_{\theta^{\prime}}$ et $S_{\rho^{\prime}}$. L'écart qui subsiste peut s'expliquer par le forçage différent en $\mathrm{d} \bar{\rho} / \mathrm{d} y$, beaucoup plus important près de la paroi pour Wardana $[10,11]$ que dans notre cas (Fig. 6). Étant donnée la correspondance qualitative entre le comportement dynamique de $\rho^{\prime}(t)$ et de la vitesse instantanée normale à la paroi $v^{\prime}(t)$, nous avons voulu confronter $S_{\rho^{\prime}}$ à la distribution $S_{v^{\prime}}$ dans une 

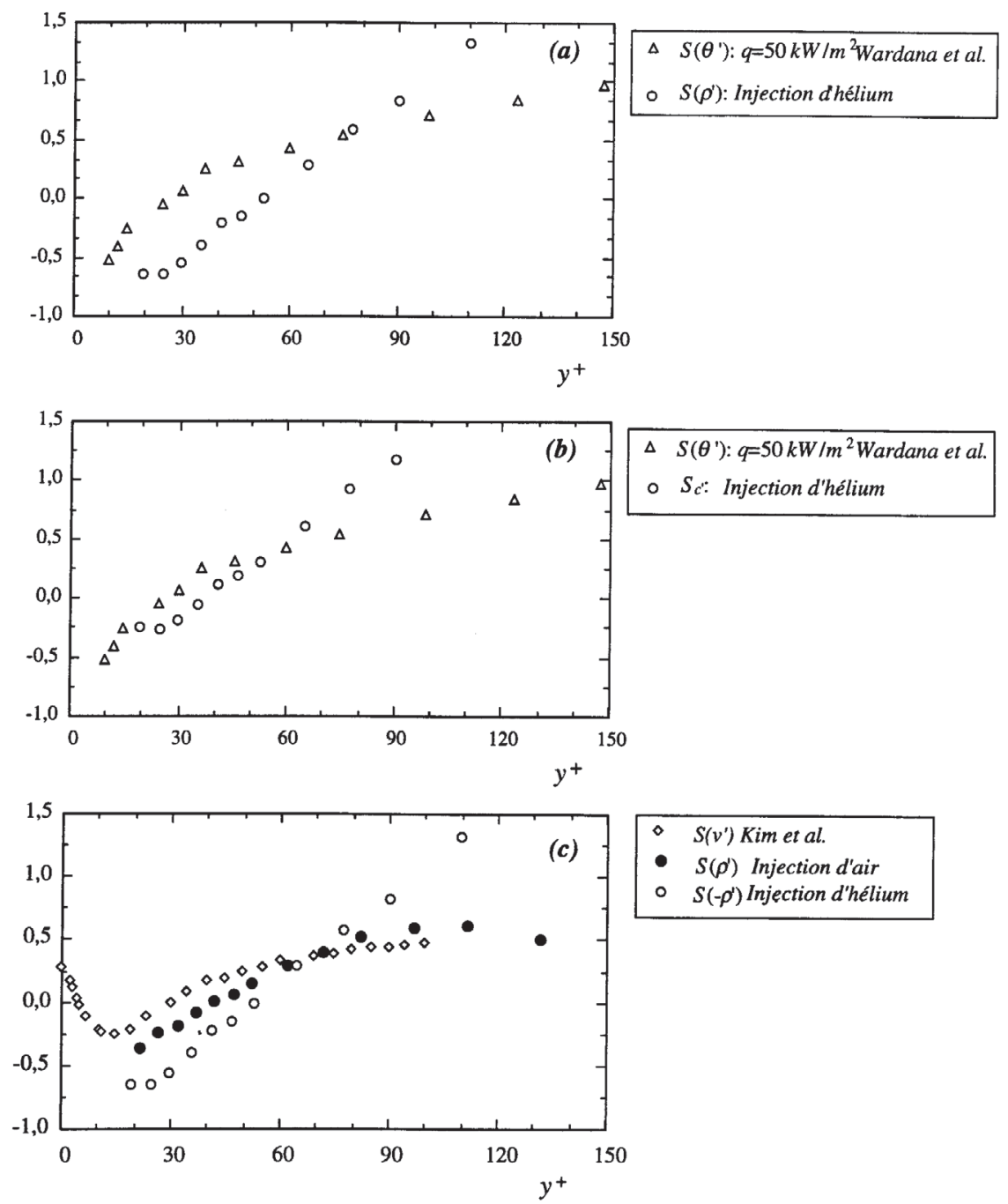

Fig. 7. Profils des facteurs de dissymétrie de vitesse, de densité et de température (pour la présente étude, $x / \delta=5$ ).

couche limite canonique. La figure $7 \mathrm{c}$ regroupe les profils de $S_{v^{\prime}}$ de Kim et al. [26], et de $S_{\rho^{\prime}}$ respectivement pour l'injection d'air et l'injection d'hélium, à $x / \delta=5$. $S_{v^{\prime}}$ coïncide d'une façon remarquable avec $S_{\rho^{\prime}}$ pour les faibles valeurs de $\partial \bar{\rho} / \partial y$ (injection d'air). L'écart entre $S_{v^{\prime}}$, dans la couche limite canonique, et $S_{\rho^{\prime}}$ à $y^{+} \leq 50$ pour l'injection d'hélium, s'explique encore une fois par l'effet direct du gradient de la masse volumique, et par l'histoire entière du développement de l'écoulement en aval de la fente d'injection.

\subsubsection{Facteurs d'aplatissement}

Les facteurs d'aplatissement $\quad F_{u^{\prime}}=\overline{u^{\prime 4}} /\left(\overline{u^{\prime 2}}\right)^{2}$ et $F_{\rho^{\prime}}=\overline{\rho^{\prime 4}} /\left(\overline{\rho^{\prime 2}}\right)^{2}$ sont liés au caractère intermittent de $u^{\prime}(t)$ et $\rho^{\prime}(t)$. Plus la valeur de $F$ est élevée, plus le signal est constitué de bouffées compactes isolées dans le temps. La figure 8 montre la distribution de $F_{u^{\prime}}$ dans la couche interne pour $x / \delta \geq 2,5, \partial \bar{\rho} / \partial y>0$ et $\partial \bar{\rho} / \partial y<0$. Hormis quelques différences subtiles, il n'y a pas d'effet du gradient de densité sur la structure intermittente de la vitesse longitudinale instantanée dans la zone développée, $x / \delta \geq 5$. Des différences subsistent bien sûr entre les profils de $F_{u^{\prime}}$ pour $\partial \bar{\rho} / \partial y>0$ et $\partial \bar{\rho} / \partial y<0$, dans la zone de relaxation où les gradients longitudinaux sont importants. Il faut cependant noter que $\partial \bar{\rho} / \partial x_{i}$ a un effet moindre sur les moments d'ordre 4 par rapport aux moments d'ordre 3 . La distribution de $F_{u^{\prime}}$ correspondant à l'injection d'hélium est confrontée aux mesures obtenues dans une couche limite canonique $(\Delta \rho=0)$ et sur une paroi fortement chauffée [11], sur la figure 9 . D'une manière générale les profils se superposent, indiquant qu'il y a seulement un effet subtil d'inhomogénéité spatiale sur l'intermittence. On remarque en outre une parfaite cohérence entre nos mesures et celles de Barlow et Johnson $(\Delta \rho=0)$ dans toute la couche limite interne. Quant aux différences par rapport au profil de $F_{u^{\prime}}$ obtenu sur une paroi chauffée, il faut être relativement vigilant car il y a une dispersion significative et une certaine incohérence dans ces mesures pour $10 \leq y^{+} \leq 30$ (Wardana et al. [11]; Fig. 9 : p. 11). Les mêmes interprétations sont approximativement valables en ce qui concerne $F_{\rho^{\prime}}$ (Fig. 10). Il y a cependant une différence quelque peu significative entre le 

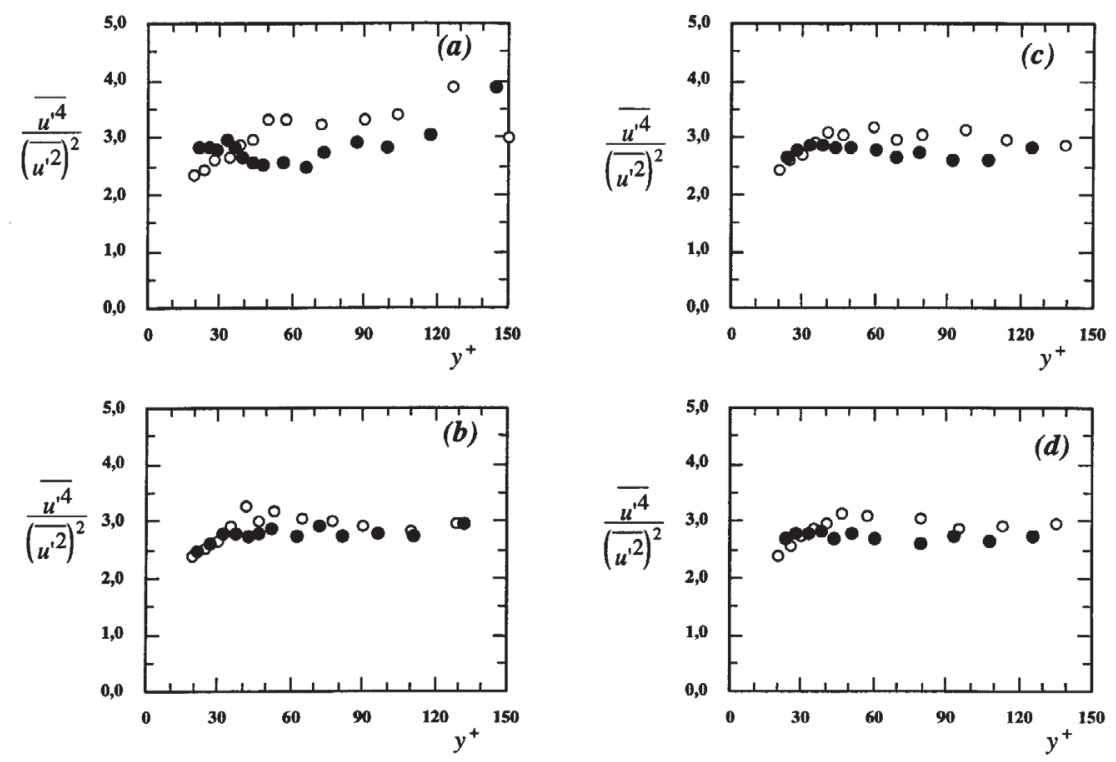

○ Injection d'air

- Injection d'hélium

\begin{tabular}{|c|c|c|c|c|}
\hline Profil & $\boldsymbol{a}$ & $\boldsymbol{b}$ & $\boldsymbol{c}$ & $\boldsymbol{d}$ \\
\hline$x / e$ & 16,67 & 33,33 & 41,67 & 50 \\
\hline$x / \delta$ & 2,50 & 5,0 & 6,25 & 7,50 \\
\hline$\Delta \rho / \rho_{\infty}=\left(\overline{\rho_{p}}-\rho_{\infty}\right) / \rho_{\infty}$ & $0,10($ Air $)$ & 0,06 & 0,050 & 0,050 \\
$0,37(\mathrm{He})$ & 0,20 & 0,200 & 0,200 \\
\hline$\left(-\left(\delta / \rho_{\infty}\right)(\partial \bar{\rho} / \partial y)\right)_{\mathrm{air}}$ & 0,50 & 0,18 & 0,13 & 0,13 \\
\hline$\left(\left(\delta / \rho_{\infty}\right)(\partial \bar{\rho} / \partial y)\right)_{\mathrm{He}}$ & 1,2 & 0,4 & 0,3 & 0,3 \\
\hline
\end{tabular}

Fig. 8. Profils du facteur d'aplatissement $F_{u^{\prime}}=\overline{u^{\prime 4}} /\left(\overline{u^{\prime 2}}\right)^{2}$.

comportement dynamique de $F_{u^{\prime}}$ et de $F_{\rho^{\prime}}$. On remarque sur la figure 10 que $\left(F_{\rho^{\prime}}\right)_{\partial \bar{\rho} / \partial y>0}$ (injection d'hélium) est systématiquement plus faible comparé avec $\left(F_{\rho^{\prime}}\right) \partial \bar{\rho} / \partial y<0$ (injection d'air) pour $10 \leq y^{+} \leq 30$. Le processus dynamique donnant lieu aux fluctuations $\rho^{\prime}(t)$, est donc légèrement moins intermittent en présence de $\partial \bar{\rho} / \partial y>0$ suffisamment fort, et ceci au début de la couche limite logarithmique.

Nous avons trouvé une concordance relativement raisonnable entre le facteur de d'aplatissement des fluctuations de température $\theta^{\prime}$ et de la concentration $c^{\prime}$ et nous présentons ces résultats sur la figure 11. La raison pour laquelle nous comparons $F_{\theta^{\prime}}$ avec $F_{c^{\prime}}$ (et non $F_{\rho^{\prime}}$ avec $F_{\theta^{\prime}}$ ), a été donnée dans la section précédente. Les différences entre $F_{\theta^{\prime}}$ et $F_{c^{\prime}}$ obtenus sur la figure 11 sont là encore, dues au gradient fort de $\partial \bar{\rho} / \partial y$ dans la sous-couche adjacente à la paroi chauffée, alors que $\partial \bar{\rho} / \partial y \sim 0$ pour $y \rightarrow 0$ dans nos expériences.

Nous terminons cette section par une remarque sur la similitude (approximative) entre les fluctuations de densité et celles de la vitesse normale à la paroi $v^{\prime}$. Il y a une bonne concordance qualitative entre $F_{v^{\prime}}$ et $F_{\rho^{\prime}}$ dans la couche tampon et au début de la couche logarithmique (Fig. 12). Cependant, on ne peut pas confirmer réellement cette similitude, car nos mesures n'ont pas pu être réalisées suffisamment près de la paroi. Le caractère frappant de $v^{\prime}(t)$ est l'augmentation spectaculaire de $F_{v^{\prime}}$ lorsque $y \rightarrow 0$, i.e. $v^{\prime}(t)$ est fortement intermittente dans la sous-couche visqueuse. C'est d'ailleurs sur ce fait qu'est basé un certain nombre de modèles de la turbulence pariétale. La comparaison entre $F_{v^{\prime}}$ et $F_{\rho^{\prime}}$ dans la zone $y^{+} \geq 0$, ne permet par conséquent pas d'affirmer d'une façon nette, que le caractère intermittent de ces quantités est similaire.

\section{Conclusion}

Dans cet article, nous avons analysé les données expérimentales des fluctuations de vitesse et de densité obtenues dans une couche limite turbulente d'un mélange air-hélium. Cette analyse apporte une contribution à la compréhension physique des mouvements des structures cohérentes dans une couche limite turbulente en présence de fortes variations de densité. Cette étude permet de dégager un certain nombre de conclusions :

- La relaxation du facteur de dissymétrie de $u^{\prime}$ s'effectue plus rapidement dans la couche externe que près de 


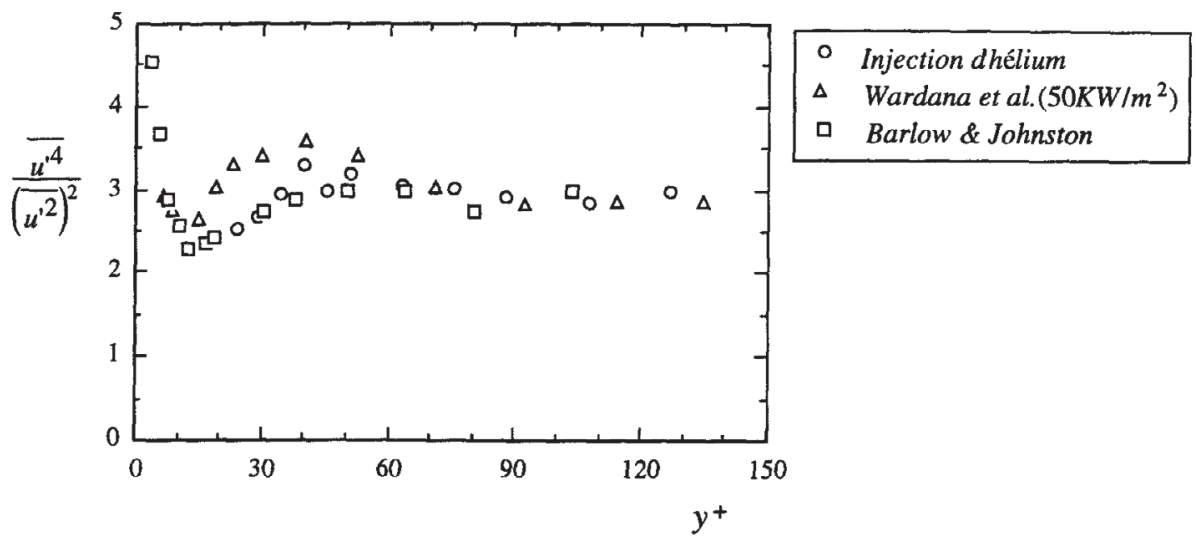

Fig. 9. Comparaison des facteurs d'aplatissement $F_{u^{\prime}}=\overline{u^{\prime 4}} /\left(\overline{u^{\prime 2}}\right)^{2}$ (pour la présente étude, $x / \delta=5$ ).
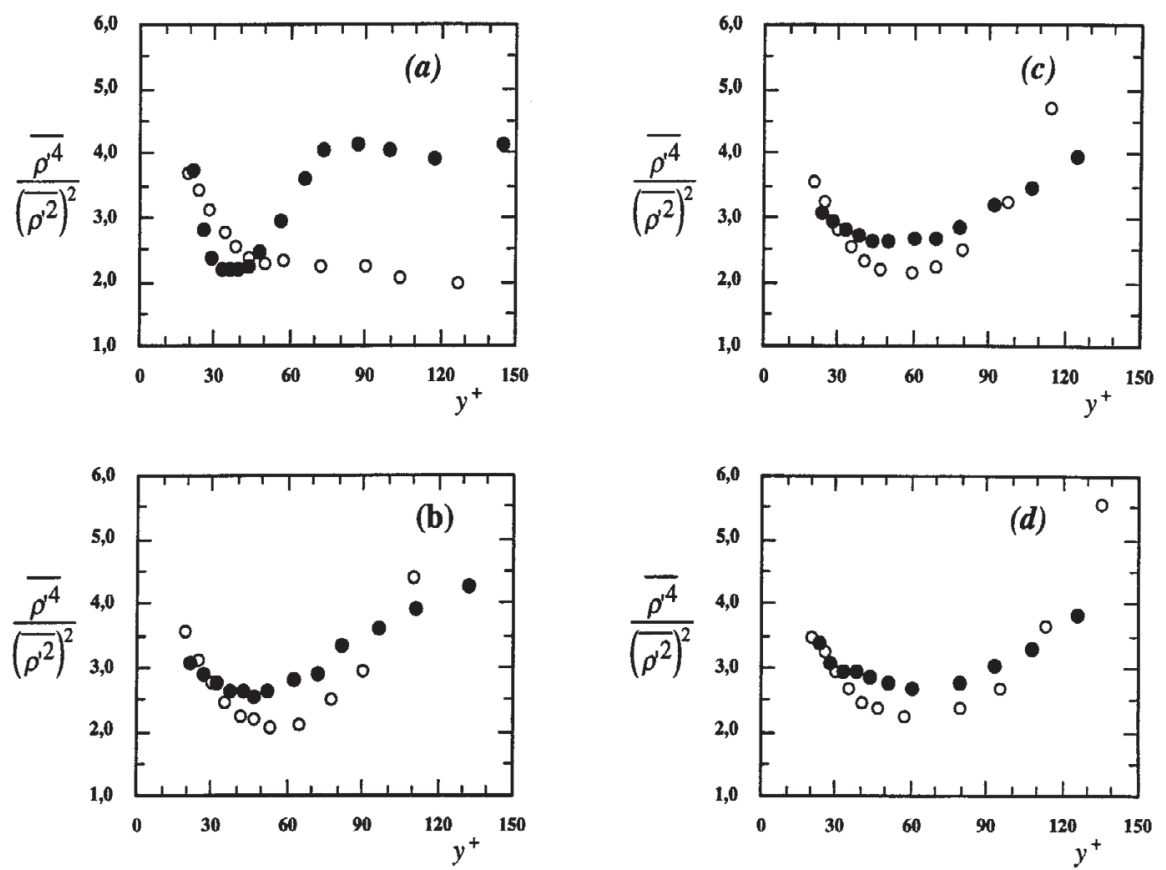

○ Injection d'air

- Injection d'hélium

\begin{tabular}{|c|c|c|c|c|}
\hline Profil & $\boldsymbol{a}$ & $\boldsymbol{b}$ & $\boldsymbol{c}$ & $\boldsymbol{d}$ \\
\hline$x / e$ & 16,67 & 33,33 & 41,67 & 50 \\
\hline$x / \delta$ & 2,50 & 5,0 & 6,25 & 7,50 \\
\hline$\Delta \rho / \rho_{\infty}=\left(\overline{\rho_{\mathrm{p}}}-\rho_{\infty}\right) / \rho_{\infty}$ & $0,10($ Air $)$ & 0,06 & 0,050 & 0,050 \\
$0,37(\mathrm{He})$ & 0,20 & 0,200 & 0,200 \\
\hline$\left(-\left(\delta / \rho_{\infty}\right)(\partial \bar{\rho} / \partial y)\right)_{\mathrm{air}}$ & 0,50 & 0,18 & 0,13 & 0,13 \\
\hline$\left(\left(\delta / \rho_{\infty}\right)(\partial \bar{\rho} / \partial y)\right)_{\mathrm{He}}$ & 1,2 & 0,4 & 0,3 & 0,3 \\
\hline
\end{tabular}

Fig. 10. Profils du facteur de dissymétrie $F_{\rho^{\prime}}=\overline{\rho^{\prime 4}} /\left(\overline{\rho^{\prime 2}}\right)^{2}$. 


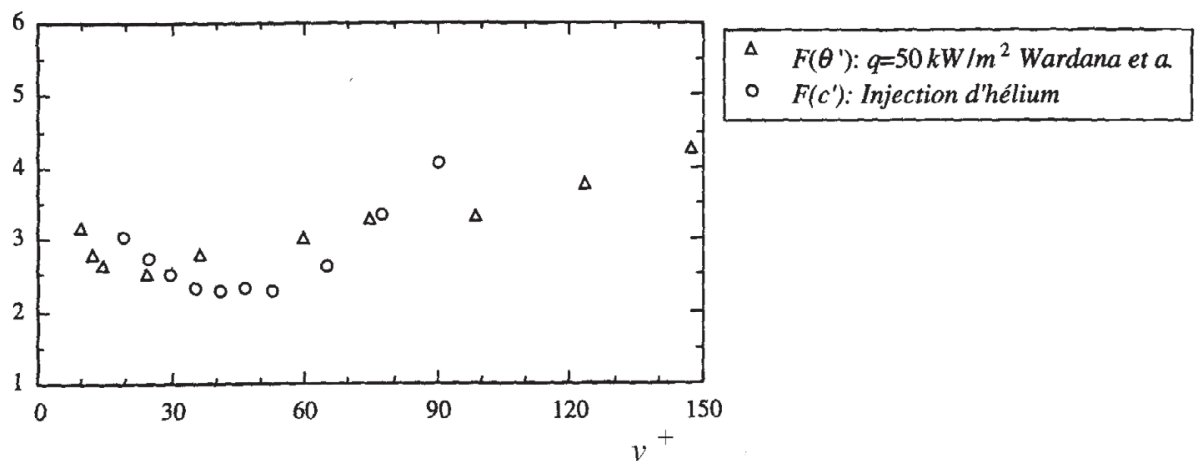

Fig. 11. Comparaison des facteurs d'aplatissement $F_{\theta^{\prime}}$ et $F_{c^{\prime}}$ (pour la présente étude, $x / \delta=5$ ).

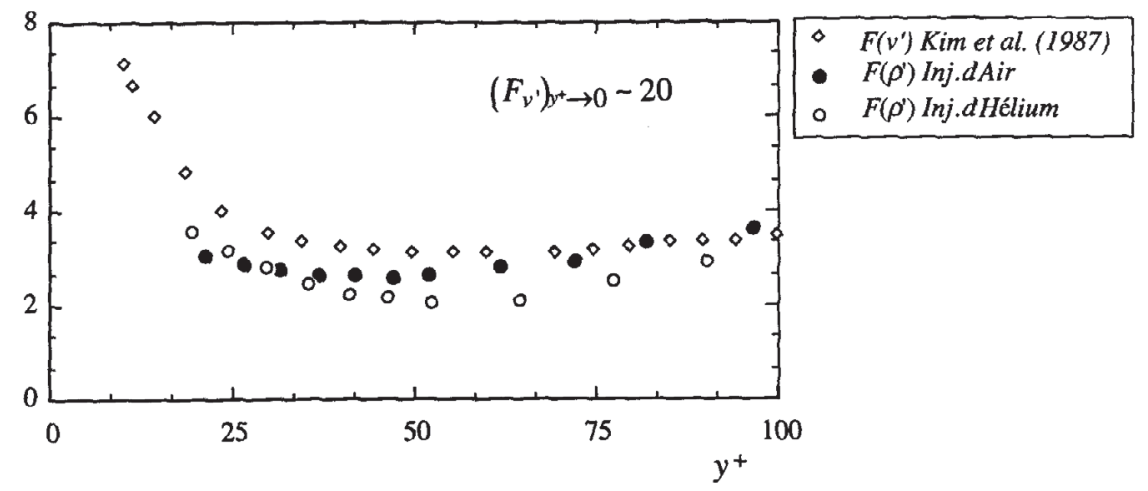

Fig. 12. Comparaison des facteurs d'aplatissement $F_{v^{\prime}}$ et $F_{\rho^{\prime}}$ (pour la présente étude, $x / \delta=5$ ).

la paroi, aussi bien pour l'injection de l'air que pour l'injection d'hélium.

- $S_{u^{\prime}}$ croît près de la fente d'injection d'une façon appréciable et cette augmentation est nettement plus accentuée pour l'injection de l'air que l'injection d'hélium. Ceci peut s'expliquer par l'augmentation du flux convectif longitudinal de l'énergie turbulente dans cette zone.

- La relaxation du facteur de dissymétrie $S_{\rho^{\prime}}$, est qualitativement similaire à celle de $S_{u^{\prime}}$, avec cependant certaines différences importantes.

- À l'exception de quelques différences subtiles, il n'y a pas d'effet du gradient de densité sur la structure intermittente de la vitesse longitudinale instantanée dans la zone développée, $x / \delta \geq 5$. Les différences qui subsistent sont bien sûr entre les profils de $F_{u^{\prime}}$ pour $\partial \bar{\rho} / \partial y>0$ et $\partial \bar{\rho} / \partial y<0$, dans la zone de relaxation où les gradients longitudinaux sont importants.

- $\partial \bar{\rho} / \partial x_{i}$ a un effet moindre sur les moments d'ordre 4 par rapport aux moments d'ordre 3 .

- Les résultats obtenus montrent aussi que la structure fine de la turbulence est significativement affectée par l'injection d'hélium dans une couche limite de gaz beaucoup plus lourd.

- Une contribution importante des éjections dans la partie externe de la couche limite et des balayages près de la paroi.

- Les résultats montrent que la région aval (jusqu'à $5 \delta$ ) est caractérisée par une lente relaxation vers le régime de la couche limite canonique. Il semble bien que les gradients de densité locaux ne contribuent plus de manière significative à l'énergie de la turbulence et que les modifications de structure de la turbulence résultent à cette distance de l'injection d'un effet de mémoire des bouleversements initiaux.

\section{Références}

[1] R.K. Cheng, T.T. Ng, Some aspects of strongly heated turbulent boundary layer flow, Phys. Fluids 25(8) (1982) 1333-1341

[2] J.L. Harion, Influence de différences de densité importantes sur les propriétés de transfert d'une couche limite turbulente, Thèse, INPG, France, 1994

[3] L. Joly, Écoulements turbulents cisaillés libres à masse volumique variable : analyse physique et modélisation, Thèse, ENSICA, France, 1994

[4] J.C. LaRue, P.A. Libby, Measurements in the turbulent boundary layer with slot injection of helium, Phys. Fluids 20(2) (1977) 192-202

[5] J.C. LaRue, P.A. Libby, Further results related to the turbulent boundary layer with slot injection of helium, Phys. Fluids 23(6) (1980) 1111-1118

[6] R.A. Antonia, Conditional sampling in turbulence measurements, Annual Rev. Fluid Mech. 13 (1981) 131

[7] K. Fukui, M. Nakajima, H. Ueda, Coherent structure of turbulent longitudinal vortices in unstably-stratified turbulent flow, Int. J. Heat Mass Transfer 34 (1991) 2373-2385 
[8] S.J. Kline, S.K. Robinson, Quasi-coherent structures in the turbulent boundary layer : Part I, Status report on a community-wide summary of the data, in : Near-Wall Turbulence, St J. Kline, N.H. Afgan (ed.), New York, Hemisphere, 1990, pp. 200-217

[9] R.F. Blackwelder, R.E. Kaplan, On the wall structure of the turbulent boundary layer, J. Fluid Mech. 76(1) (1976) 89-112

[10] I.N.G. Wardana, T. Ueda, M. Mizimoto, Velocity - temperature correlation in strongly heated channel flow, Exp. Fluids 18 (1995) 454-461

[11] I.N.G. Wardana, T. Ueda, M. Mizimoto, Structure of turbulent two-dimensional channel flow with strongly heated wall, Exp. Fluids 13 (1992) 17-25

[12] D.G. Bogard, W.G. Tiederman, Burst detection with single point velocity measurements, J. Fluid Mech. 162 (1986) 389-413

[13] E.R. Corino, R.S. Brodkey, A visual study of turbulent shear flow, J. Fluid Mech. 37 (1969) 1-30

[14] H.T. Kim, S.J. Kline, W.C. Reynolds, The production of turbulence near a smooth wall in a turbulent boundary layer, J. Fluid Mech. 50(1) (1971) 133-160

[15] S.S. Lu, W.W. Willmarth, Measurements of the structure of the Reynolds stress in a turbulent boundary layer, J. Fluid Mech. 60(3) (1973) 481-511

[16] W.W. Willmarth, S.S. Lu, Structure of the Reynolds stress near the wall. J. Fluid Mech. 55 (1972) 65-92

[17] J. Cousteix, R. Houdeville, Turbulence and skin friction evolution in an oscillating boundary layer,
Fifth Symposium on Turbulent Shear Flows, Cornell University, 1985

[18] S.K. Robinson, Coherent motions in the turbulent boundary layer, Annu. Rev. Fluid Mech. (23) (1991) 601-639

[19] J.M. Wallace, R.S. Brodkey, H. Eckelmann, Patternrecognized structures in boundary shear flows, J. Fluid Mech. 83 (1977) 673-684

[20] J. Way, P.A. Libby, Application of hot wire anemometry and digital techniques to measurements in a turbulent helium jet, A.I.A.A.J. 9(8) (1971) 1567-1573

[21] J. Way, P.A. Libby, Hot wire probes for measuring velocity and concentration in helium-air mixtures, A.I.A.A.J. 8(5) (1970) 976-978

[22] R.K. Cheng, T.T. Ng, Conditional Reynolds stress in a strongly heated turbulent boundary layer with premixed combustion, Phys. Fluids 28(2) (1985) 473488

[23] A. Bejan, Convective heat transfer, John Wiley \& Sons, New York, 1995

[24] L.P. Purtell, P.S. Klebanoff, F.T. Buckley, Turbulent boundary layer at Low Reynolds number, Phys. Fluids 24(5) (1981) 802-811

[25] P. Dangel, H.H. Fernholz, M. Hess, Effects of longitudinal diffusion in the fluid and of heat transfer conduction to the substrate on the response of hot film gages, in Springer-Verlag, editor, Advances in turbulence, 1987, pp. $470-479$

[26] H.T. Kim, P.R. Moin, R. Moser, Turbulences statistics in fully developed channel flow at low Reynolds number, J. Fluid Mech. 177 (1987) 133-166

Retrouvez nos articles sur le site : www.edpsciences.org/meca 